\title{
Anesthesia Implications of the Use of Essential Oils in Alzheimer's Dementia
}

\author{
James L Geiger* \\ Medical Advisory Panel, doTERRA, USA
}

*Corresponding author: Dr. James L Geiger, MD, Medical Advisory Panel, doTERRA, USA, E-mail: jlgeiger@oilmd.com

\begin{abstract}
The diagnosis and treatment of Alzheimer's dementia (AD) has gained much attention due to the current and predicted prevalence of the disease. The biomarkers of neural inflammation, oxidative stress, genetics and the multiple medical etiologies of $A D$ coupled with the poly-pharmacy of comorbid conditions and diseases associated with AD are complex. The medical diagnosis and treatments of AD need more reliability and integrative health regimens, such as supplementation with generally regarded as safe (GRAS) essential oils that could be implemented clinically to lessen the global economic impact to societies, and disruptions to families.

Treatment has focused on the management of $A D$ based on the cholinergic theory attempting to prevent cognitive decline while preserving short-term memory with prescription medications. Certain essential oils exhibiting acetylcholinesterase inhibition (ACHEI) activity derived from natural phyto-preparations might also be utilized in the setting of the decreasing memory of $A D$. Prescription psychotropic medications might be therapeutically interchanged to certain essential oils to manage behavioral and psychiatric issues associated with $A D$. Advancing age and anesthesia increase the risk of post-operative cognitive decline (POCD) associated with common procedures and surgeries that $A D$ patients commonly undergo. Globally, plant material preparations such as extracts and essential oils are analyzed for anti-inflammatory, anti-infectious and anti-anxiety, anti-depressive, analgesic and ACHEI activity.

The potential for use of GRAS essential oils as supplements for seniors in the prevention and treatment of the cognitive decline and behavioral disruptions of aging should be studied to determine the safe and effective methods of inhalational, topical and ingestion aromatherapy techniques. The landmark literature reviewed here explores the mechanisms and synergy of the aromatic sciences that can provide the basis for designing and implementing protocols for the integration of GRAS essential oils into the wide variety of clinical situations encountered in the aging population, with and without $A D$.
\end{abstract}

\section{Keywords}

Alzheimer's Dementia, Memory, Anxiety, Aromatherapy, Acetylcholinesterase, Anesthesia, Essential oils, Lavender, Ginger

\section{Key Subjects}

Natural Products, Phytotherapy, Biochemistry, Acetylcholinesterase, Healthcare

\section{Introduction}

Alzheimer's dementia (AD) is characterized by impairment of memory and progressive neurodegeneration resulting in profound cognitive dysfunction. The social, clinical and financial implications of $A D$ are remarkable in view of the fact that the prevalence of $A D$ is expected to double every five years [1]. Dementia can be of mixed pathology yet AD prevalence is about $60 \%$, followed by vascular dementia (VD) $20 \%$ and dementia with Lewy bodies $10 \%$ (DLB) [1]. There are frontotemporal dementias (FTD) and other sub-types perhaps related to stroke, decreased cerebral blood flow, concussion, traumatic brain injury or congestive heart failure [2].

The mainstay of initial treatment of memory loss is with anticholinesterase inhibitors (ACHEI) and can be coupled with non-pharmaceutical integrative health regimens such as music therapy, bright light therapy, ultrasound, massage and aromatherapy with generally regarded as safe essential oils (GRAS). The progressive nature of $A D$ can lead to behavioral and psychological symptoms of dementia (BPSD) including neuroses such as agitation, anxiety, panic attacks, aggression, depression and psychoses requiring neuroleptic prescriptions [3].

Citation: Geiger JL (2018) Anesthesia Implications of the Use of Essential Oils in Alzheimer's Dementia. Int J Anesthetic Anesthesiol 5:065. doi.org/10.23937/2377-4630/1410065

Accepted: May 23, 2018: Published: May 25, 2018

Copyright: (c) 2018 Geiger JL. This is an open-access article distributed under the terms of the Creative Commons Attribution License, which permits unrestricted use, distribution, and reproduction in any medium, provided the original author and source are credited. 
The cholinergic theory of memory improvement in $A D$ rests on the pharmacologic principles of synaptic acetylcholine acting on nicotinic and muscarinic receptors as it applies to neurological and musculoskeletal biochemistry and physiology. Acetylcholine esterase is the principle enzyme involved in the hydrolysis of acetylcholine that is important for synaptic transmission of neurochemical signals for memory and muscle strength. Prescriptions of anticholinesterase inhibitor (ACHEI) medications prolong the ability of acetylcholine to remain in the synaptic cleft, resulting in the mainstay of memory loss prevention yet they neither delay nor reverse the progressive nature of $A D$.

Donepezil is a synthetic ACHEI medication approved in the late 1990's for conditions of mild to moderate memory loss. Donepezil antagonizes the effects of non-depolarizing muscle relaxants and it is recommended to be discontinued two weeks before some surgeries. Rivastigimine was approved in 2000 having been designed from a natural alkaloid, the anesthesia medication, physostigmine. Discoveries of ACHEI alkaloid chemical activity have been made in marine, fungal and plant kingdoms showing the complex activities of the following classes of molecules: indoles, quinolones and piperidines [4].

A natural alkaloid, huperzine A, found in Huperzia spp. is an example of traditional Chinese herbal medicine (TCM). There are other examples of TCM, such as Gingko biloba and Panax ginseng providing notable memory improvement when synergistically paired together [5]. The non-alkaloid classes of ACHEI of significance are flavonoids, phenolics and terpenoids, which are also known as isoprenoids.

\section{The Aromatic Science of Essential Oils in Alz- heimer's Dementia}

Plants produce a wide variety of aromatic volatile organic compounds (VOC) for either protection from predators or to attract pollinators or seed dispersers. The largest groups of plant volatiles are terpenes that are found in multiples of five carbon units, monoterpenes, diterpenes and sesquiterpenes. These terpenes are synthesized in chloroplasts along the methylerythritol pathway and in the cytoplasm along the mevalonate pathway. The VOC from plants when manufactured into essential oils derived from specific parts of plants have the ability to alter the expression or suppression of plant, animal and human organism's genetic makeup [6].

Research in vitro on numerous steam distilled commercially available essential oils (GRAS), such as basil, tarragon, lavender, Spanish sage, tea tree and rosemary have been shown to exert varied degrees of inhibition of acetylcholinesterase activity. Similar testing of single chemical constituents such as 1,8-cineole, alpha pinene, eugenol, alpha terpinol and terpien-4-ol have ACHEl ef- fects but to a lesser degree [7]. There are significant differences in ACHEl activities between whole plant essential oils and single chemical constituents implying that there are synergies and possibly antagonisms perhaps generated by secondary messenger chemical constituents [8].

Butyrylcholinesterase (BCHE), also known as pseudocholinesterase, has genetic variability in humans potentially impacting anesthesia negatively, causing prolonged paralysis when low plasma levels of BCHE prevent the normally rapid hydrolysis of the normally short acting neuromuscular depolarizing agent, succinylcholine, consisting of two acetylcholine molecules, often used for emergency tracheal intubation. The ability to recover from respiratory muscle paralysis and regain adequate return of respiratory muscle strength may take 6-12 hours, requiring temporary mechanical ventilatory support depending on the degree of expression of inherited autosomal recessive genes.

Laboratories in many countries have shown that essential oils have significant activity as ACHEl and butyrylcholinesterase inhibition (BCHEI). Some plant research has determined that BCHE has significant response to inhibition by various essential oils and specific single chemical constituents of many essential oils from plants. It is significant that essential oils obtained from whole plant material containing the complete spectrum of constituents produced from specific parts of plants were more active inhibitors of $\mathrm{ACHEI}$ and $\mathrm{BCHEI}$ than single plant volatile constituents [9].

Essential oils of lemon, eucalyptus globulus (EG), cypress and thyme have both high antioxidant values and notably high ACHEl activity as well. A significant finding is that even though EG was the most effective ACHEI, the major constituents of EG, limonene and 1-8-cineole were not responsible for the high level ACHEI activity, while d-3-carene more actively inhibited acetylcholinesterase [10].

Active sites of acetylcholine promote the deposition of $A ß$ plaques in the brain of $A D$ patients. It has been determined that both $\mathrm{ACHEI}$ and $\mathrm{BCHEI}$ molecules prevent the formation of $A ß$ plaques connecting their functions synergistically with multi-target capabilities. The use of major natural alkaloid phytochemical compounds can be exploited as newly synthesized molecules for further study for possible treatment of other neurodegenerative diseases such as Parkinson's disease and the muscle disease, myasthenia gravis [11]. The four stages of plant cellular biosynthesis of terpenoids are actively being evaluated for pharmaceutical properties that may be synthesized for numerous medical indications such as analgesics and anti-inflammatory agents by genetically engineering and manufacturing single chemical constituents using bacterial cells as factories, for example the prokaryotic cell, E. coli. [12]. 
Monoterpenes are representative of up to $90 \%$ of the aromatic chemistry of essential oils and are structured as acyclic monoterpenes, monocyclic monoterpenes and bicyclic monoterpenes acting by multiple mechanisms on a vast variety of receptors too numerous and complex to mention here. Acyclic monoterpenes usually modulate the opioid system and monocyclic monoterpenes modulate analgesia without the participation of the opioid system. In general, most monoterpenes act to suppress the chemical expression of inflammatory mediators by inhibiting the activity of the cyclooxygenases (COX) resulting in reduced prostaglandin induced inflammation [13].

Many monoterpenes have been studied in vitro examining activity on both the acute and chronic mediators of inflammation. In part, the immuno-stimulatory actions of essential oils are due to the blockade of receptors and chemical pathways of pro-inflammatory mediators such as the leukotrienes via 5-lipooxgenase as a result of the known actions of complete essential oils from specific parts of plants and single chemical constituents [14].

Current anesthesia literature speaks to the neural control of inflammation and the cholinergic inflammatory reflex as a therapeutic target in medicine for sepsis, ventilator-induced lung injury, myocardial ischemia, traumatic hemorrhage, and POCD [15]. Perioperatively the practice of administration of non-depolarizing neuromuscular blocking medications for specific surgical indications is routinely reversed with acetylcholinesterase inhibitors to prevent residual neuromuscular paralysis in the post-anesthesia care unit (PACU). The neural inflammatory reflex can be managed with medications such as atropine an antimuscarinic, glycopyrrolate an anticholinergic agents and the FDA approved AD treatment, galantamine, an ACHEl that is centrally acting, activating the efferent arm of the inflammatory reflex [15]. It is not known how much supplementation with essential oils or single chemical constituents would be required to raise acetylcholine levels to achieve the same degree of positive effect on memory as when taking a prescription medication.

\section{Integrative Allopathic and Complementary Health- care: Synergy with Essential Oils}

The interactions of two or more chemical constituents from numerous essential oils have been shown to produce a combined effect greater than the sum of effects generated by a single constituent. The effects produced are substantially greater when the complete plant essential oil, distilled from a specific plant part is tested over and above that of the individual plant's chemical constituents e.g. oxygenated monoterpenes. The synergist effects and benefits of the chemical constituents from plants and whole essential oils from some known part of a plant has been described in animal and human studies some of which are randomized, placebo controlled trials (RCT) [16].
Numerous clinical scenarios have identified natural materials and integrated protocols using phyto-pharmaceuticals into the medical management of pain, nausea and the challenging diseases associated with specialties, such as infectious diseases, surgery and anesthesia [17]. Synergism has been identified between various natural plant products, essential oils and antibiotics to combat bacterial, fungal and mycobacterial diseases [18]. Multiple modes of actions of specific chemical constituents, such as carvacrol, eugenol, thymol, cinnamaldehyde and cinnamic acid against multiple targets have been described showing that these phytochemicals enhance the activity of antibiotics [19]. These findings are becoming more evident and useful clinically in the management of drug resistant, multi-drug resistant organisms and "super-bug" diseases [20].

Synergy of anti-microbial effects between constituents of essential oils or whole essential oils and antibiotics mixed with essential oils and nano-particles has been demonstrated [21]. Synergistic effects exist between linalool and alpha-terpineol when combined with ampicillin and kanamycin [22]. Essential oils of anise, ginger, chamomille, hyssop, thyme and sandalwood have anti-viral properties exhibiting a clear virucidal activity against Herpes simplex type 2, interacting at the viral envelope [23].

Synergy exists between surgery and anesthesia since both specialties present numerous challenges including pain, nausea and cognitive dysfunction especially in $A D$ that have been addressed with protocol-based applications of phyto-supplementation with essential oils [2426]. Post-operative nausea and vomiting (PONV) is managed with a multi-modal drug therapy approach [27]. Drugs of various categories, even those with black box warnings, including antihistamines, anti-emetics, tranquilizers and the hypnotic propofol have been utilized as an anesthesia technique to prevent PONV due to the synergism between medications and the positive effects exerted on multiple receptors [28].

Ondansetron works via the vagus nerve and serotonergic pathway. Integrating prophylactic intravenous multimodal therapy with the essential oil of ginger, Zingiber officinale, in the acute care and ambulatory setting to prevent the general anesthesia complication of PONV significantly increases successful outcomes [29]. The question of patient satisfaction after surgery is influenced by PONV outcomes. Essential oils used in an anesthesia RCT study to prevent nausea after surgery utilized either ginger alone or a blend of ginger spearmint, peppermint and cardamom placed on a 2 -inch by 2 -inch gauze. Patients were instructed to inhale nasally for five minutes. This methodology was shown to be effective treatment of postoperative nausea (PON) in the PACU [30].

Lifestyle factors that include exercise and proper diet have a positive effect of delaying late life $A D$ gen- 
otype expression [31]. Neural memory and disordered awareness of memory loss (i.e., anosognosia) occurs along both serotonergic and cholinesterase chemical pathways. There are certain stages of functional memory loss that have shown improved management by the antagonism of glutaminergic neurotransmission at $\mathrm{N}$-Methyl-D-Aspartate (NMDA) receptors with memantine [31].

There is general agreement that medical regimens for $A D$ with anticholinergic agents should be minimized in those with $A D$, especially before prescribing cholinergic medications [3]. There are strong evidence based studies recommending treatments for AD presented in this review of RCTs; memantine for moderate to severe $A D$ in combination with $\mathrm{ACHEI}, \mathrm{ACHEI}$ are not effective in FTD and may cause agitation, selective serotonin receptor inhibitor (SSRI) medications may help behavioral but not cognitive issues, no drugs are clearly effective in VD, though ACHEI may be effective in mixed dementias [3]. Statins, anti-inflammatory agents and supplementations with vitamin $\mathrm{E}$ were not recommended as effective anti-dementia agents in this consensus statement from the British Association for Psychopharmacology [3].

\section{Metabolomics to Measure Effects of Supple- ments}

Older adults are known to ingest various supplements. In one review study of non-essential oils supplements in people aged 50-76 found there was no association for the majority of supplements with total mortality, only glucosamine and chondroitin were each associated with decreased total mortality [32]. Supplementation with essential oils nutrigenomically has been documented in the laboratory and some human models to modulate genomics and has anti-inflammatory, analgesic, immunomodulatory, anticancer, hepatoprotection, hypolipidemic, anti-diabetic, antioxidant bone reparation, antidepressant, and $\mathrm{ACHEI}$ properties [33].

The therapeutic molecular mechanisms of essential oils and application of aromatherapy techniques for the treatment of brain serotonin and dopamine based emotions known for management of anxiety and depression is associated with significant benefits and low risk [34]. The 5-hydroxytryptophan system forms the basis for prescription antidepressants, antipsychotics and anxiolytics enhancing neurotransmitter synthesis and decreasing breakdown pathways of norepinephrine and dopamine. These same pathways are stimulated by VOC scents of essential oils such as lavender, lemon and bergamot that impact olfactory chemical-electrical-chemical pathways to brain nuclei that influence mood and behavior positively [34].

A metabolomic study was performed on 31 healthy young human females with mild anxiety and their urine specimens analyzed after ten days of inhalation of essential oils of 4 aromatic plants: Lavandula angustifo- lia, Salvia sclarea, Santalum album and Citrus sinensis. Significant alterations of metabolic profile was characterized by increased levels of arginine, homocysteine, and betaine as well as decreased levels of alcohols, carbohydrates, and organic acids in urine and that some participants had marked variation in metabolomic profile while others did not have much variance [35]. The metabolisms of the tricarboxylic cycle and gut microbial metabolism were also influenced. The changes observed could be related to food intake and individual responsiveness to inhalation of specific VOC [35].

Considering intestinal dysbiosis, the following essential oils Carum carvi, Lavendula angustifolia, Trachyspermum copticum, and Citrus aurantium essential oils displayed the greatest degree of selectivity, inhibiting the growth of potential pathogens at concentrations in vitro that had no effect on the beneficial bacteria examined [36]. Metabolic analysis of rat's brain and urine metabolites initially identified increased carbohydrates, fatty acids and lowered amino acids. These changes in metabolism were significantly reduced after 10 days of aroma inhalation along with the anxiety produced on the elevated plus maze (EPM) [37]. Anxiety often causes insomnia especially in AD and there are numerous positive studies showing the low risk and significant benefits of inhaled applications of essential oils such as lavender, jasmine, juniper, basil and marjoram to enhance sleep quantity and quality while reducing anxiety [38].

Essential oil of lavender angustifolia in 318 male and female participants aged 18-68 with ICD-10 diagnostic criteria for mixed anxiety-depression orally ingesting 80 mg per day was found to be efficacious and effective [39]. Topically applied essential oils in diluted concentrations of essential oils of melissa and lavender angustifolia has been shown to have safe and effective benefits in several clinical settings in an analysis of various RCTs on aromatherapy treatments provided for patients with behavioral and psychological symptoms of dementia [16]. The Committee on Herbal Medicinal Products in the European legislation defines phytopharmaceutical preparations as complex multicomponent mixtures that contain several active ingredients acting on multiple targets [16]. Lavender angustifolia, $80 \mathrm{mg}$ capsules taken orally acts as an anxiolytic by potent inhibition of voltage-dependent calcium channels without binding to gabapentin binding sites and by significant reduction of serotonin receptor binding potential [16].

Topically applied aromatherapy with essential oil of Melissa officinalis was found to provide superior quality of life when compared to donepezil in terms of neuropsychiatric symptoms in this RCT study of 114 patients, mean age of 85, in which the essential oil of melissa was applied in a cream during a massage twice a day for 12 weeks [16]. When considering drug-essential oils interactions it is notable that the cytochrome P450 enzymatic pathway of drug metabolism in healthy volunteers 
had no clinically relevant inhibitory or inducing effects on caffeine, tolbutamide, omeprazole, midazolam or dextromethorphan, a NMDA receptor antagonist, when $160 \mathrm{mg}$ of lavender angustifolia in repeated doses was administered orally [40].

\section{Responsible, Safe and Effective Applications of Essential Oils}

There are numerous medical challenges encountered during aging with AD. A review of systems is often notable for blood pressure management that is critical to avoid vascular accidents such as strokes resulting in dysphagia possibly leading to the occurrence of aspiration. The scent of black pepper is a strong appetite stimulant. In older patients in this Japanese study of carefully selected patient's suffering from stroke with swallowing dysfunction, black pepper essential oils inhalation resulted in improvement of reflexive swallowing movement regardless of level of consciousness or physical or mental activity, possibly by activation of the insular and orbital frontal cortex [41]. Respiratory reactive airway diseases may progress to bronchitis or pneumonias from various causes. The immune system can be stimulated by inhaled vapors of essential oil of eucalyptus [42]. The cellular protective effects of essential oils of orange peel, clove bud, cinnamon leaf, cinnamon bark, eucalyptus leaf and rosemary leaf was studied in vitro using influenza $A$ virus (IAV) infecting Madin-Darby canine kidney cells (MDCK) demonstrating a positive concentration dependent effect against IAV and lack of toxicity to MDCK while attenuating and inhibiting IAV protein synthesis at the post-transcription level [43].

Oral ingestion of a chemical constituent of many essential oils, 1-8 cineole, also known as eucalyptol, which works as an expectorant and anti-inflammatory agent. This study has shown significant respiratory benefits as defined by an Asthma Quality of Life questionnaire in 247 asthmatics studied over 6 months, requiring lowered steroid dose for control of symptoms when ingesting $600 \mathrm{mg}$ of 1-8 cineole in capsules in divided dose per day, [44]. The monoterpene, 1,8-cineole commonly found in eucalyptus globulus, is a known mucolytic and spasmolytic of the respiratory tract due to the anti-oxidative and anti-inflammatory properties via action on interleukin and T-cell derived cytokines [45]. Cineole has beneficial use as long-term therapy in prevention of chronic obstructive pulmonary disease exacerbations and improved asthma control where $200 \mathrm{mg}$ of cineole was found to be equal to $3.8 \mathrm{mg}$ of prednisolone in this review citing current evidence for co-medication with cineole in inflammatory airway diseases [45].

The genitourinary tract may develop recurrent urinary tract infections, formation of renal, ureteral or bladder stones and possible renal insufficiency or failure. Rosemary and basil essential oils have been analyzed in a hospital microbiology laboratory to inhibit growth of approximately 60 strains of $E$. coli [46]. Advancing ar- thritis, loss of motor strength and disequilibrium associated with increased risks of falls, may require surgery for total joint replacement or hip/shoulder hemiarthroplasty. The positive effect of inhaled $3 \%$ concentration on $4 \times 2$ inch gauze of eucalyptus essential oil on three consecutive days post-operatively was determined to decrease the pain and decrease diastolic blood pressure after total knee replacement when using continuous passive motion as shown by Visual Analogue Pain Scale, in 25 of 27 patients ranging in age from 43 to 85 and of 68.2 mean age [47]. The probability of developing cancer that requires anesthesia and surgery during a lifetime increases with age [48]. Negative effects of volatile anesthesia gas and narcotics on the immune system may play a role in cancer reoccurrence [49].

A cancer diagnosis encountered early or as part of end of life care in AD is a dilemma. Light Thai massage in 94 patient's, ages 30-70 with colorectal cancer, after 7-10 day course of chemotherapy that were enrolled in a one week RCT study with $0.5 \mathrm{ml}$ of ginger essential oil in coconut oil had improved cellular immunity as measured by CD4/CD8 ratios and improved self-rated symptom severity scores [50]. These components of essential oils commonly occur in food as eucalyptol, eugenol, borneol, carvacrol and thymol have differing anti-oxidative, anti-mutagenic and anti-carcinogenic properties in the laboratory [51]. There is a role for natural phenolic compounds from essential oils, in particular eugenol, in cancer chemoprevention via the regulation of the cell cycle [52]. The integration of human clinical aromatic science trials and results of laboratory studies suggest indications with implications for humans that needs further elucidation in the use of the plant based GRAS essential oil supplements for seniors.

\section{Conclusion}

There are many reasons and clinical conditions to integrate the use of essential oils in the early and end of life care of the elderly and patients with dementia including AD. The amount of literature depicting the safe and effective uses of GRAS essential oils by aromatic inhalation, low concentration topical application and ingestion is significant. Integrative healthcare is becoming more prevalent; including the use of aromatherapy services on patients with acute and chronic conditions in the acute care setting [53]. Administration of specially designed aromatherapy protocols based on North American Nursing Diagnosis Association (NANDA) diagnoses, implemented with policies and procedures with specific guidelines that includes consent for aromatherapy and "time out" to verify allergies are warranted. The aromatherapy plan and results of treatment could be documented in the patient's electronic medical record (EMR) generating metrics e.g. patient satisfaction and quality improvement outcomes for future analysis.

\section{References}

1. Hebert LE, Beckett LA, Scherr PA, Evans DA (2001) An- 
nual incidence of Alzheimer disease in the United States projected to the years 2000 through 2050. Alzheimer Dis Assoc Disord 15: 169-173.

2. Meng L, Hou W, Chui J, Han R, Gelb AW (2015) Cardiac output and cerebral blood flow: the integrated regulation of brain perfusion in adult humans. Anesthesiology 123: 11981208.

3. O'Brien JT, Holmes C, Jones M, Jones R, Livingston G, et al. (2017) Clinical practice with anti-dementia drugs: A revised (third) consensus statement from the British Association for Psychopharmacology. J Psychopharmacol 31: 147-168.

4. Dey A, Bhattacharya R, Mukherjee A, Pandey DK (2017) Natural products against Alzheimer's disease: Pharmaco-therapeutics and biotechnological interventions. Biotechnol Adv 35: 178-216.

5. May BH, Feng M, Zhou IW, Chang SY, Lu SC, et al. (2016) Memory impairment, dementia, and alzheimer's disease in classical and contemporary traditional chinese medicine. J Altern Complement Med 22: 695-705.

6. Zitzelsberger C, Buchbauer G (2015) Essential oils as "A Cry for Help”. A review. Nat Prod Commun 10: 1127-1138.

7. Dohi S, Terasaki M, Makino M (2009) Acetylcholinesterase inhibitory activity and chemical composition of commercial essential oils. J Agric Food Chem 57: 4313-4318.

8. Savelev S, Okello E, Perry NS, Wilkins RM, Perry EK (2003) Synergistic and antagonistic interactions of anticholinesterase terpenoids in Salvia lavandulaefolia essential oil. Pharmacol Biochem Behav 75: 661-668.

9. Orhan I, Kartal M, Kan Y, Sener B (2008) Activity of essential oils and individual components against acetyl- and butyrylcholinesterase. Z Naturforsch C 63: 547-553.

10. Aazza S, Lyoussi B, Miguel MG (2011) Antioxidant and antiacetylcholinesterase activities of some commercial essential oils and their major compounds. Molecules 16: 7672-7690.

11. Shah AA, Dar PA, Ganie SA, Kamal MA, Dar TA (2017) A current perspective on the inhibition of cholinesterase by natural and synthetic inhibitors. Curr Drug Metab 18: 96111.

12. Lange BM, Ahkami A (2013) Metabolic engineering of plant monoterpenes, sesquiterpenes and diterpenes--current status and future opportunities. Plant Biotechnol J 11: 169196.

13. Guimarães AG, Quintans JS, Quintans LJ Jr (2013) Monoterpenes with analgesic activity--a systematic review. Phytother Res 27: 1-15.

14. de Cássia da Silveira e Sá R, Andrade LN, de Sousa DP (2013) A review on anti-inflammatory activity of monoterpenes. Molecules 18: 1227-1254.

15. Steinberg BE, Sundman E, Terrando N, Eriksson LI, Olofsson PS (2016) Neural control of inflammation: implications for perioperative and critical care. Anesthesiology 124: 1174-1189.

16. Press-Sandler O, Freud T, Volkov I, Peleg R, Press $Y$ (2016) Aromatherapy for the treatment of patients with behavioral and psychological symptoms of dementia: A descriptive analysis of RCTs. J Altern Complement Med 22: 422-428.

17. Lakhan SE, Sheafer H, Tepper D (2016) The effectiveness of aromatherapy in reducing pain: A systematic review and meta-analysis. Pain Res Treat 2016: 8158693.
18. Hemaiswarya S, Kruthiventi AK, Doble M (2008) Synergism between natural products and antibiotics against infectious diseases. Phytomedicine 15: 639-652.

19. Langeveld WT, Veldhuizen EJ, Burt SA (2014) Synergy between essential oil components and antibiotics: A review. Crit Rev Microbiol 40: 76-94.

20. Boire NA, Riedel S, Parrish NM (2013) Essential oils and future antibiotics: New weapons against emerging 'Superbugs'? J Anc Dis Prev Rem 1: 105.

21. Mahendra Rai, Priti Paralikar, Priti Jogee, Gauravi Agarkar, Avinash P Ingle, et al. (2017) Synergistic antimicrobial potential of essential oils in combination with nanoparticles: Emerging trends and future perspectives. Int J Pharm 519: 67-78.

22. Mossa JS, El-Feraly FS, Muhammad I (2004) Antimycobacterial constituents from Juniperus procera, Ferula communis and Plumbago zeylanica and their in vitro synergistic activity with isonicotinic acid hydrazide. Phytother Res 18: 934-937.

23. Koch C, Reichling J, Schneele J, Schnitzler P (2008) Inhibitory effect of essential oils against herpes simplex virus type 2. Phytomedicine 15: 71-78.

24. Sears SR, Bolton S, Bell KL (2013) Evaluation of "Steps to Surgical Success" (STEPS): A holistic perioperative medicine program to manage pain and anxiety related to surgery. Holist Nurs Pract 27: 349-357.

25. Stea S, Beraudi A, De Pasquale D (2014) Essential oils for complementary treatment of surgical patients: State of the art. Evid Based Complement Alternat Med 2014: 726341.

26. Kapila AK, Watts HR, Wang T, Ma D (2014) The impact of surgery and anesthesia on post-operative cognitive decline and Alzheimer's disease development: Biomarkers and preventive strategies. J Alzheimers Dis 41: 1-13.

27. Mandal P, Das A, Majumdar S, Bhattacharyya T, Mitra T (2014) The efficacy of ginger added to ondansetron for preventing postoperative nausea and vomiting in ambulatory surgery. Pharmacognosy Res 6: 52-57.

28. Wax D, Doshi A, Hossain S, Bodian CA, Krol M (2007) Changing patterns of postoperative nausea and vomiting prophylaxis drug use in an academic anesthesia practice. $J$ Clin Anesth 19: 356-359.

29. James L Geiger (2005) The essential oil of ginger, Zingiber officinale, and anesthesia. The International Journal of Aromatherapy 15: 7-14.

30. Hunt R, Dienemann J, Norton HJ, Hartley W, Hudgens A, et al. (2013) Aromatherapy as treatment for postoperative nausea: A randomized trial. Anesth Analg 117: 597-604.

31. Deak F, Kapoor N, Prodan C, Hershey LA (2016) Memory loss: Five new things. Neurol Clin Pract 6: 523-529.

32. Pocobelli G, Kristal AR, Patterson RE, Potter JD, Lampe $\mathrm{JW}$, et al. (2010) Total mortality risk in relation to use of less-common dietary supplements. Am J Clin Nutr 91: 1791-1800.

33. Cayuela Sánchez JA, Elamrani A (2014) Nutrigenomics of essential oils and their potential domestic use for improving health. Nat Prod Commun 9: 1641-1648.

34. Lv XN, Liu ZJ, Zhang HJ, Tzeng CM (2013) Aromatherapy and the central nerve system (CNS): therapeutic mechanism and its associated genes. Curr Drug Targets 14: 872-879.

35. Yinan Zhang, Yani Wu, Tianlu Chen, Lei Yao, Jiajian Liu, et al. (2013) Assessing the metabolic effects of aromathera- 
py in human volunteers. Evid Based Complement Alternat Med 2013: 356381.

36. Hawrelak JA, Cattley T, Myers SP (2009) Essential oils in the treatment of intestinal dysbiosis: A preliminary in vitro study. Altern Med Rev 14: 380-384.

37. Wu Y, Zhang Y, Xie G, Zhao A, Pan X, et al. (2012) The metabolic responses to aerial diffusion of essential oils. PLoS One 7: e44830.

38. Lillehei AS, Halcon LL (2014) A systematic review of the effect of inhaled essential oils on sleep. J Altern Complement Med 20: 441-451.

39. Kasper S, Volz HP, Dienel A, Schläfke S (2016) Efficacy of Silexan in mixed anxiety-depression--A randomized, placebo-controlled trial. Eur Neuropsychopharmacol 26: 331340.

40. Doroshyenko O, Rokitta D, Zadoyan G, Klement S, Schläfke S, et al. (2013) Drug cocktail interaction study on the effect of the orally administered lavender oil preparation silexan on cytochrome P450 enzymes in healthy volunteers. Drug Metab Dispos 41: 987-993.

41. Ebihara $T$, Ebihara $S$, Maruyama $M$, Kobayashi M, Itou $A$, et al. (2006) A randomized trial of olfactory stimulation using black pepper oil in older people with swallowing dysfunction. J Am Geriatr Soc 54: 1401-1406.

42. Sadlon AE, Lamson DW (2010) Immune-modifying and antimicrobial effects of Eucalyptus oil and simple inhalation devices. Altern Med Rev 15: 33-47.

43. Wu S, Patel KB, Booth LJ, Metcalf JP, Lin HK, et al. (2010) Protective essential oil attenuates influenza virus infection: An in vitro study in MDCK cells. BMC Complement Altern Med 10: 69.

44. Worth H, Dethlefsen U (2012) Patients with asthma benefit from concomitant therapy with cineole: a placebo-controlled, double-blind trial. J Asthma 49: 849-853.
45. Juergens UR (2014) Anti-inflammatory properties of the monoterpene 1.8-cineole: Current evidence for co-medication in inflammatory airway diseases. Drug Res (Stuttg) 64: 638-646.

46. Sienkiewicz $M$, Łysakowska $M$, Pastuszka M, Bienias $W$, Kowalczyk E (2013) The potential of use basil and rosemary essential oils as effective antibacterial agents. Molecules 18: 9334-9351.

47. Jun YS, Kang P, Min SS, Lee JM, Kim HK, et al. (2013) Effect of eucalyptus oil inhalation on pain and inflammatory responses after total knee replacement: A randomized clinical trial. Evid Based Complement Alternat Med 2013: 502727.

48. Hamidpour R, Hamidpour S, Hamidpour M, Shahlari M, Sohraby M (2014) Summer savory: From the selection of traditional applications to the novel effect in relief, prevention, and treatment of a number of serious illnesses such as diabetes, cardiovascular disease, alzheimer's disease, and cancer. J Tradit Complement Med 4: 140-144.

49. Kim R (2016) Anesthetic technique and cancer recurrence in oncologic surgery: Unraveling the puzzle. Cancer Metastasis Rev 36: 159-177.

50. Khiewkhern S, Promthet S, Sukprasert A, Eunhpinitpong W, Bradshaw P (2013) Effectiveness of aromatherapy with light thai massage for cellular immunity improvement in colorectal cancer patients receiving chemotherapy. Asian Pac J Cancer Prev 14: 3903-3907.

51. Slamenova D, Horvathova E (2013) Cytotoxic, anti-carcinogenic and antioxidant properties of the most frequent plant volatiles. Neoplasma 60: 343-354.

52. Jafari S, Saeidnia S, Abdollahi M (2014) Role of natural phenolic compounds in cancer chemoprevention via regulation of the cell cycle. Curr Pharm Biotechnol 15: 409-421.

53. Johnson JR, Rivard RL, Griffin KH, Kolste AK, Joswiak D, et al. (2016) The effectiveness of nurse-delivered aromatherapy in an acute care setting. Complement Ther Med 25: 164-169. 